\title{
ДАВЛЕНИЕ НАСЫЩЕННОГО ПАРА МОНОХЛОРИДА ИНДИЯ ПО ДАННЫМ СПЕКТРОФОТОМЕТРИИ И НУЛЬ-МАНОМЕТРИИ
}

\author{
(ㄷ 2019 А. Ю. Завражнов*, А. В. Наумов, Е. Н. Малыгина, А. В. Косяков \\ Воронежский государственный университет \\ Университетская пл. 1, 394018 Воронеж, Российская Федерация
}

\begin{abstract}
Аннотация. Измерены спектры поглощения пара монохлорида индия, находящегося в состояниях насыщенного и ненасыщенного пара относительно расплава InCl в присутствии расплава металлического индия. Спектры исследованы в интервале длин волн $200-400 \mathrm{~nm}$ и диапазоне температур $225-850^{\circ} \mathrm{C}$. Показано, что в этих условиях пар состоит из молекул $\mathrm{InCl}$ и в пределах чувствительности эксперимента не содержит других молекулярных форм хлоридов индия. В ходе нуль-манометрического эксперимента найдена температурная зависимость $\ln p_{\text {InCl }}=-A / T+b$ давления насыщенного пара в трехфазном равновесии $L_{\mathrm{In}}-L_{\mathrm{InCl}}-V$, параметры которой составили: $A=-10255 \pm 69 \mathrm{~K}, b=1095 \pm 0.08$ (давление - относительно стандартного $1 \mathrm{~atm}$ ). Показано, что угловой коэффициент $A$ хорошо согласуется с угловым коэффициентом температурной зависимости коэффициента поглощения $\ln T k(\lambda)=-A / T+B(\lambda)$ при различных длинах волн. Это позволяет рассматривать высокотемпературную спектрофотомерию пара как альтернативу прямому манометрическому эксперименту. При сопоставлении манометрических и спектрофотометрических данных определены значения молярного коэффициента экстинкции InCl в ненасыщенном паре для максимумов полос поглощения. Найдено, что этот коэффициент слабо линейно зависит от температуры, убывая или возрастая на разных длинах волн.
\end{abstract}

Ключевые слова: монохлорид индия, насыщенный пар, гетерогенное равновесие, высокотемпературная спектрофотометрия, тензиметрия, манометрия.

\section{ВВЕДЕНИЕ}

Равновесия с участием галогенидов индия и галлия имеют большое значение при создании новых каркасно-канальных структур, содержащих кластеры металла (типа $\mathrm{In}_{5}^{7+}$ ) [1], для разработки новых источников света $[2,3]$, а также для глубокой очистки соответствующих простых веществметаллов. Вместе с тем обращает на себя внимание тот факт, что если галлий, как и алюминий, может быть легко пересублимирован в ходе галогенидного химического транспорта $[4,5]$, то с индием аналогичный перенос затруднен [6-8]. В работах [68] эти затруднения связывали с тем, что равновесия различных галогенидных форм индия в паре с металлическим индием сильно смещены в сторону низшего галогенида InHal $(\mathrm{Hal}=\mathrm{Cl}, \mathrm{Br}, \mathrm{I})$. При этом другие молекулярные формы, необходимые для газотранспортного переноса галогенидов индия, практически отсутствуют в паре при любых температурах и давлениях.

$\triangle$ Завражнов Александр Юрьевич, e-mail: alzavr08@rambler.ru
Целью настоящего цикла работ является анализ состава пара хлоридов индия, находящегося в гомогенном и гетерогенных равновесиях с фазами системы In-Cl. В качестве основного метода исследования была выбрана УФ-спектрофотометрия паровой фазы, которая позволяет определять содержание и парциальные давления равновесных молекулярных форм. Однако применение такого метода возможно лишь в случае хорошо известных величин молярных экстинкций молекулярных форм, находящихся в паре. Поэтому основной задачей настоящей работы является нахождение коэффициентов молярной экстинкции монохлорида индия в паре, а также проверка предположений работ $[7,8]$, согласно которым в равновесии металлического индия с паром хлорида индия значительно преобладающей молекулярной формой является $\mathrm{InCl}$.

\section{ЗАДАЧИ ЭКСПЕРИМЕНТА}

Указанную задачу решали в два этапа. На nервом этапе при помощи оригинальной спектрофотометрической установки для высокотемпературных 
исследований получали спектры пара хлорида индия, который находился в трехфазном равновесии

$$
L_{\mathrm{In}}-L_{\mathrm{InCl}}-V \text {, }
$$

где $L_{\mathrm{In}}$ - расплав металлического индия, $L_{\mathrm{InCl}}-$ pacплав монохлорида индия, а $V$ - пар. При этом считали, что: $a$ ) растворимость хлора и (или) хлоридов индия в металлическом индии, равно как растворимость индия в расплаве $L_{\text {InCl }}$ пренебрежимо мала; б) пренебрежимо мало в условиях эксперимента также парциальное давление индия $p_{\text {In }}$ в паре. Это исследование позволило найти при различных температурах коэффициенты оптического поглощения $k(\lambda, T)$. Значения $k(\lambda, T)$ определялись для максимумов полос поглощения, при этом проверяли закон Бэра в форме:

$$
k(\lambda, T)=\varepsilon(\lambda, T) C_{\mathrm{InCl}}(T)
$$

или

$$
k(\lambda, T)=\varepsilon_{p}(\lambda, T) p_{\mathrm{InCl}}(T),
$$

где $\varepsilon$ - молярная экстинкция, зависящая, вообще говоря, от температуры; $\varepsilon_{p}-$ экстинкция в расчете на единицу парциального давления; $C_{\mathrm{Incl}}$ и $p_{\mathrm{InCl}}-$ молярная объемная концентрация и парциальное давление монохлорида индия, зависящие от температуры в случае пара, насыщенного относительно жидкой фазы $L_{\mathrm{InCl}}$ Учитывалась также возможность отклонения от закона Бэра, когда $\varepsilon$ (или $\varepsilon_{p}$ ) начинают зависеть от концентрации (или парциального давления).

Кроме того был организован эксперимент, в котором наблюдалась смена вариантности равновесия. В этом опыте количество расплава хлорида индия бралось настолько малым, что эта фаза полностью испарялась при некоторой температуре. Тогда при превышении данной температуры трехфазное равновесие (1) сменялось двухфазным

$$
L_{\text {In }}-V \text {. }
$$

Исследование этого равновесия позволило проследить за температурной зависимостью коэффициентов поглощения в условиях, когда концентрация монохлорида индия в паре оставалась постоянной, т. е. в условиях, когда эта зависимость могла быть связана только с собственным температурным изменением молярной экстинкции $\mathrm{InCl}$.

Определение коэффициентов молярной экстинкции стало возможным после выполнения второго этапа работы, связанного манометрическими исследованиями. Задачей этих исследование было прямое измерение давления насыщенного пара монохлорида индия в зависимости от температуры.

\section{МЕТОДИКА СПЕКТРОФОТОМЕТРИЧЕСКОГО ЭКСПЕРИМЕНТА}

В спектрофотометрическом исследовании исследуемое равновесие реализовывали в герметичной кварцевой кювете, имеющей форму параллелепипеда $(25 \times 10 \times 10 \mathrm{~mm})$ с толщиной поглощающего слоя $10.00 \mathrm{~mm}$. В кювету вводили металлический индий и очень небольшие количества монохлорида индия. Вакуумированную и отпаянную кювету помещали в вертикальную печь, снабженную небольшими окнами, пропускающими излучение. Спектры поглощения исследовали однолучевым методом при помощи монохроматора МДР-41 в диапазоне длин волн от 200 до $400 \mathrm{~nm}$. В качестве образца сравнения использовали идентичную пустую вакуумированную кювету. Подробнее схема спектрофотометрического эксперимента и применяемое при этом оборудование описаны в [9].

Подчеркнем, что в наших исследованиях вся кювета находилась в условиях, максимально близких к изотермическим. Это отличает нашу методику от схемы оптико-тензиметрических исследований Бребрика [10]. В экспериментах [10] та область кюветы, где реализовывалось гетерогенное равновесие, находилась при одной температуре $T_{1}$ (она могла варьироваться), в другой же области кюветы, где фиксировались спектры поглощения пара, поддерживалась другая, бо́льшая температура $T_{2}$, которая сохранялась неизменной на протяжении всего эксперимента. Такая схема позволяла избегать влияния температуры на экстинкцию компонентов пара, однако имела недостатки, связанные с неизотермичностью исследуемой системы.

Равновесия (1) и (2) исследовали в интервале температур 225-850 ${ }^{\circ} \mathrm{C}$. Абсорбционные спектры фиксировали в волновом диапазоне 200-800 nm с шагом 0.01 или $0.05 \mathrm{~nm}$. Предельный разрешаемый спектральный интервал составлял $0.02 \mathrm{~nm}$. Использовались режимы как пошагового увеличения, так и пошагового уменьшения температуры с шагом $20-25^{\circ} \mathrm{C}$. Время изотермических выдержек составляло от 2 до 9 часов, а критерием установления равновесия служило воспроизведение (в пределах погрешности) измеренных величин $k$ при возвращении к данной температуре от условий равновесия при других температурах. 


\section{РЕЗУЛЬТАТЫ \\ СПЕКТРОФОТОМЕТРИЧЕСКОГО ЭКСПЕРИМЕНТА}

При исследовании равновесия (1) полосы поглощения на спектрах начинали проявляться при температурах выше $215{ }^{\circ} \mathrm{C}$ (рис. 1). Полученные спектры поглощения (рис. 1a) характеризуются:
- группой интенсивных узких близко расположенных полос в волновом диапазоне от 262 до $280 \mathrm{~nm}$ с наибольшим поглощением при $267.0 \mathrm{~nm}$;

- группой также узких, но более слабых и редких полос в диапазоне длин волн 338-365 nm;

- пологой полосой с максимумом поглощения при длине волны около $208 \mathrm{~nm}$.
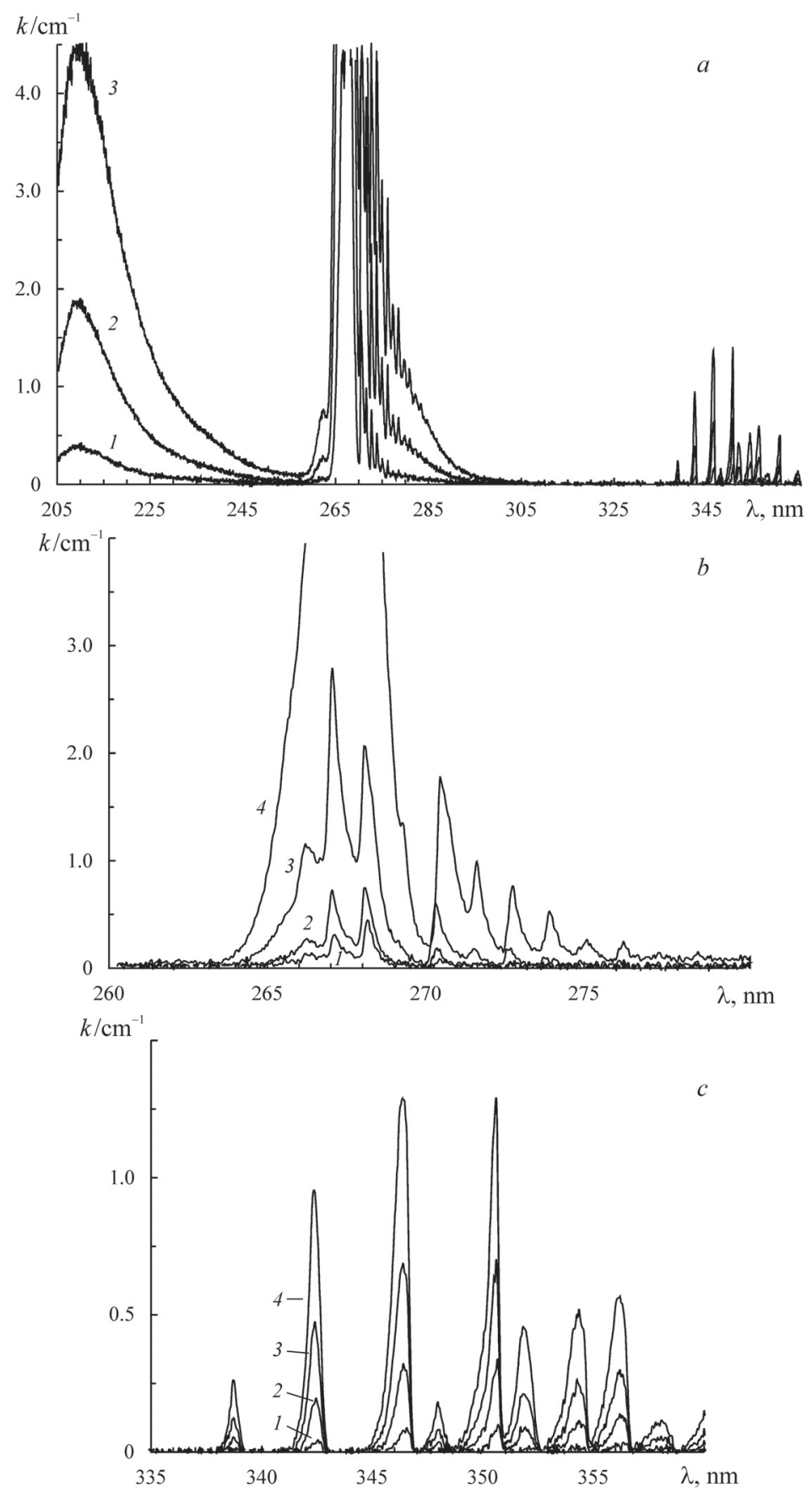

Рис. 1. Спектры поглощения пара хлорида индия в равновесии (1) при различных температурах: $a-$ общий вид спектра $\left(1-340 ; 2-400 ; 3-440{ }^{\circ} \mathrm{C}\right) ; b-$ фрагмент $260-280 \mathrm{~nm}\left(1-230 ; 2-250 ; 3-290 ; 4-340{ }^{\circ} \mathrm{C}\right)$; $c$ - фрагмент $335-360 \mathrm{~nm}\left(1-340 ; 2-400 ; 3-440 ; 4-480^{\circ} \mathrm{C}\right)$

[Fig. 1. The absorption spectra of indium chloride vapor in equilibrium (1) at different temperatures: $a-$ general view $\left(1-340 ; 2-400 ; 3-440{ }^{\circ} \mathrm{C}\right) ; b$ - range $260-280 \mathrm{~nm}\left(1-230 ; 2-250 ; 3-290 ; 4-340{ }^{\circ} \mathrm{C}\right) ; c-$ range $335-360 \mathrm{~nm}$ $\left.\left(1-340 ; 2-400 ; 3-440 ; 4-480{ }^{\circ} \mathrm{C}\right)\right]$ 
Согласно данным [10-14] первые две группы полос возникают за счет присутствия в паре молекул $\mathrm{InCl}$ и обусловлены переходами $C^{1} \Pi_{1}-X^{1} \Sigma_{0}^{+}$для первой группы $[12,13]$ и $B^{3} \Pi_{1}-X^{1} \Sigma_{0}^{+}$и $A^{3} \Pi_{0}-X^{1} \Sigma_{0}^{+}-$ для второй группы полос [14]. Интенсивность всех этих пиков возрастает при повышении температуры, что коррелирует с представлением об увеличении концентрации монохлорида индия в паре с температурой в равновесии (1). При этом последний пик с максимумом поглощения около $208 \mathrm{~nm}$ характеризуется таким же относительным увеличением интенсивности с ростом температуры, как и пики из первой группы (262-280 nm). Например, величина $k$, измеренная при $215.0 \mathrm{~nm}$ для плеча обсуждаемой полосы поглощения, при любых температурах оказывается практически совпадающей с величиной $k$ для острой полосы $273.9 \mathrm{~nm}$ из волнового диапазона 262-280 nm. Это указывает на то, что полоса с максимумом около $208 \mathrm{~nm}$ также принадлежит молекулярной форме InCl. Такое отнесение соответствует и работе [14], в которой видимо эту же полосу с максимумом поглощения при $210.1 \mathrm{~nm}$ относят к переходам $E-X$.

С учетом того, что характерные для $\mathrm{In}_{2} \mathrm{Cl}_{4}(242$ и $315 \mathrm{~nm}$ [11]) и $\mathrm{InCl}_{3}(216 \mathrm{~nm}$ [11]) полосы поглощения в наших экспериментах не наблюдались, мы полагали, что молекулярная форма $\mathrm{InCl}$ значительно преобладает над другими формами. Это подтверждается и тем, что в неизотермической системе с равновесием (2) не происходит транспортно-химический перенос индия [7] (для последнего необходимы, по меньшей мере, две молекулярные формы с различным содержанием галогена).

Ход типичной температурной зависимости коэффициента поглощения в равновесиях (1) и (2) приведен на рис. 2 на примере полосы поглощения с максимумом $270.4 \mathrm{~nm}$. Кривая $a c$ относится к трехфазному равновесию (1). На этом же графике приведена кривая abef, которая соответствует эксперименту со сменой вариантности равновесия. В этом опыте количество расплава на основе $\mathrm{InCl}$ было настолько мало, что весь монохлорид индия при некоторой температуре был полностью переведен в паровую фазу. Смена характера фазового равновесия происходит в точке $b$ : участок кривой $a b$ соответствует равновесию (1), а участок $b e f-$ равновесию (2). Изменение величины $k$ с ростом температуры на переходном участке $b e$ может быть обусловлено двумя причинами: переходом монохлорида индия из раствора на основе металла в паровую фазу при заметной растворимости $\mathrm{InCl}$ в фазе $L_{\mathrm{In}}$, либо заметной адсорбцией $\mathrm{InCl}$ на внутренних

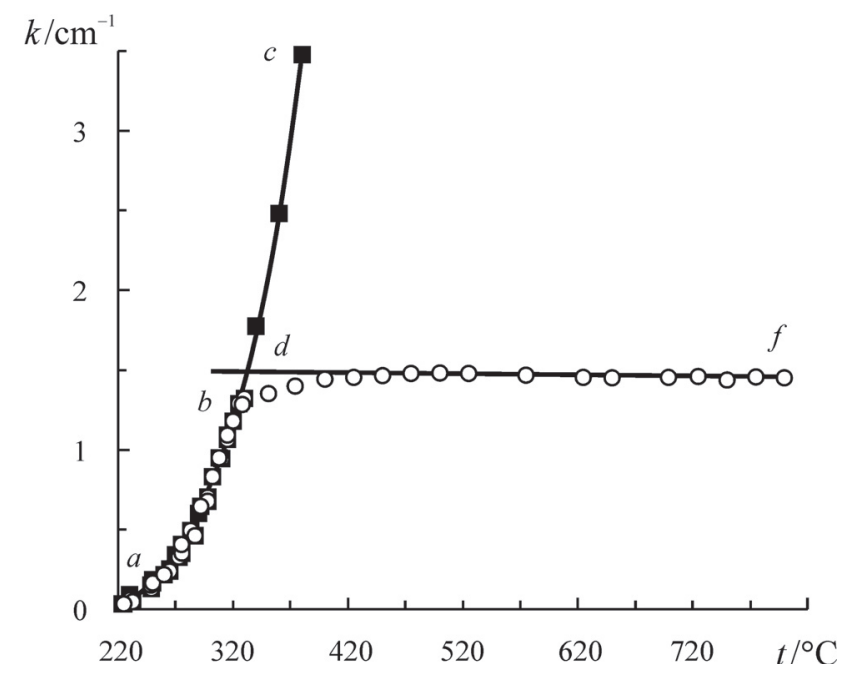

Рис. 2. Температурная зависимость коэффициента поглощения на длине волны $270 \mathrm{~nm}: a b c$ - равновесие (1); bef- равновесие (2); abef - ход зависимости в эксперименте со сменой вариантности равновесия $(1) \rightarrow(2)$

[Fig. 2. The temperature dependence of the absorption coefficient for the band $270 \mathrm{~nm}$ : $a b c$ - equilibrium (1); bef - equilibrium (2); abef - dependence with change of equilibrium $(1) \rightarrow(2)]$

стенках кварцевой кюветы. В противном случае в точке $d$ существовал бы излом.

При температуре выше $\sim 450{ }^{\circ} \mathrm{C}$ возрастание коэффициента поглощения прекращается. С учетом этого обстоятельства мы полагали, что в данном эксперименте уже при температуре $450{ }^{\circ} \mathrm{C}$ весь $\mathrm{InCl}$ оказывается в паре. Более того, на участке $e f$ ( $\left.450-850{ }^{\circ} \mathrm{C}\right)$ коэффициент поглощения незначительно убывает, что свидетельствует о температурной зависимости величины $k$ при фиксированной концентрации $\mathrm{InCl}$ в паре.

Следует заметить, что аналогичный рис. 2 вид температурных зависимостей $k(\lambda)$ для равновесий (1) и (2) был установлен и для других максимумов полос поглощения, относящихся к пару монохлорида индия. При этом в области равновесия ненасыщенного (относительно $L_{\mathrm{InCl}}$ ) пара хлорида индия, т. е. для равновесия (2), величина $k(\lambda)$ в ряде случаев слабо убывает с ростом температуры (линии 1 и 2 рис. 3), в ряде случаев - слабо возрастает (линии 3 и 4). По-видимому, такое поведение непосредственно отражает температурную зависимость экстинкции $\varepsilon(\lambda, T)$. Как видно из рис. 2,3 , эта зависимость оказывается линейной.

Наиболее подробные данные получены нами для равновесия (1). При сравнении температурных зависимостей величин $k(\lambda)$ с литературными данными по давлению насыщенного пара $p_{\mathrm{InCl}}$ может 


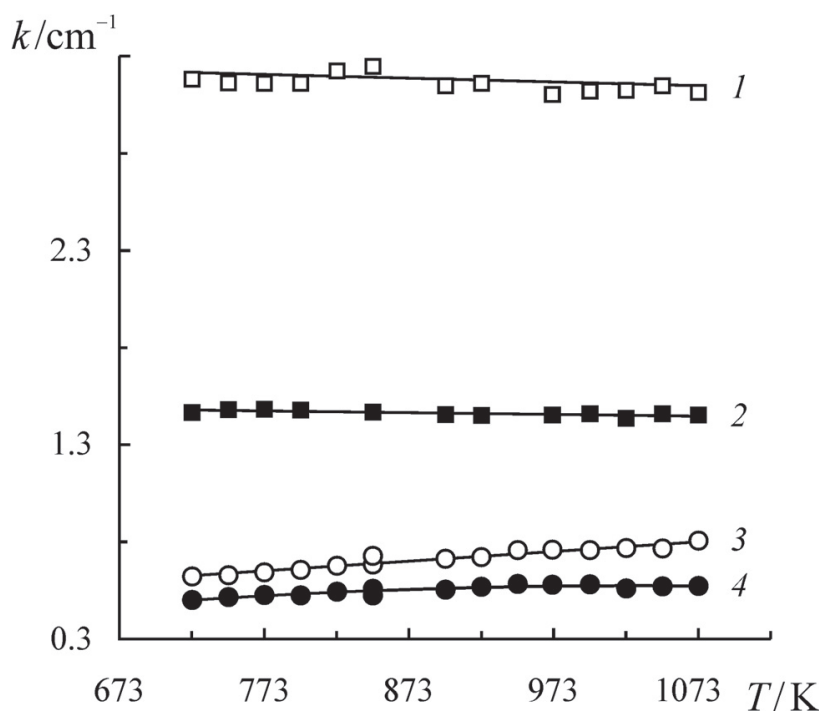

Рис. 3. Температурные зависимости коэффициента поглощения в равновесии (2) для максимумов полос поглощения: 1 - 267.0; 2 - 270.4; 3 - 271.6; 4 - $272.6 \mathrm{~nm}$

[Fig. 3. The temperature dependences of the absorption coefficient in equilibrium (2) for the maxima of absorption bands: $1-267.0 ; 2-270.4 ; 3-271.6 ; 4-272.6 \mathrm{~nm}]$

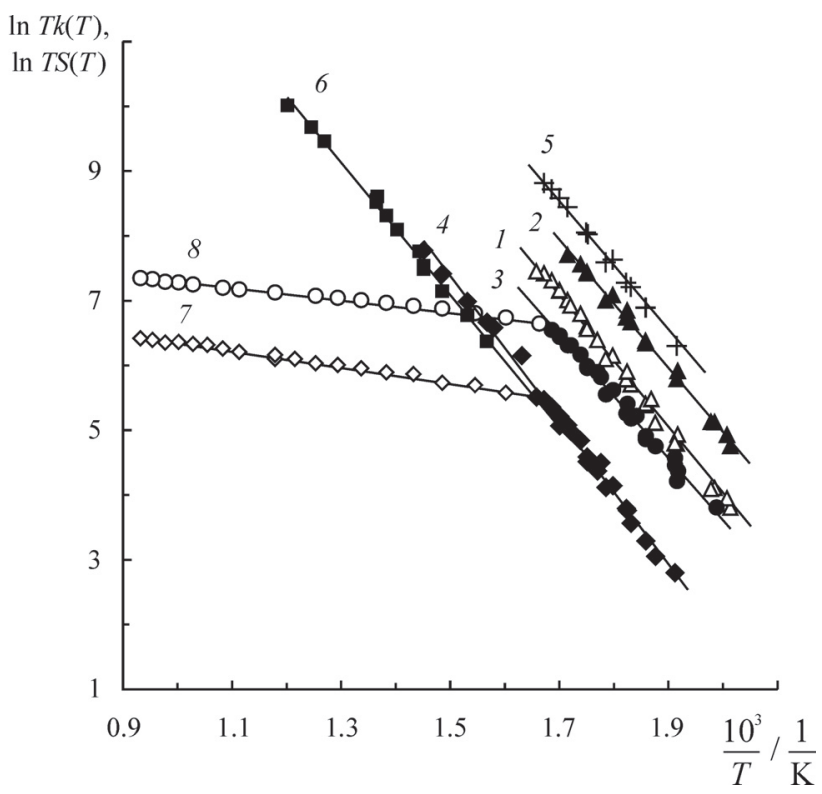

Рис. 4. Температурные зависимости величин $k(\lambda, T)$ (линии $1-4)$ и $S\left(\lambda_{1}, \lambda_{2} ; T\right)$ (линии 5,6$)$ в равновесии (1) для длин волн: $1-266.2 ; 2-267.0,3-270.4,4-$

$272.6 \mathrm{~nm} ; 5$ - интервал 262 - 280; 6 - интервал $337-365 \mathrm{~nm}$. Температурные зависимости величины $k(\lambda, T)$ со сменой характера равновесия (1) $\rightarrow(2)$ (линии 7,8) для длин волн: $7-272.6 ; 8-270.4 \mathrm{~nm}$

[Fig. 4. The temperature dependences of $k(\lambda, T)$ (lines 1 4 ) and $S\left(\lambda_{1}, \lambda_{2} ; T\right)$ (lines 5, 6 ) in equilibrium (1) for wavelengths: 1 - 266.2; 2 - 267.0, 3-270.4, 4$272.6 \mathrm{~nm} ; 5$ - range $262-280 ; 6$ - range $337-365 \mathrm{~nm}$. The temperature dependences of $k(\lambda, T)$ with change of equilibrium $(1) \rightarrow(2)$ (lines 7,8 ) for the bands: $7-272.6$; $8-270.4 \mathrm{~nm}]$ быть оценена корректность спектрофотометрических экспериментов. Такая оценка может быть проведена даже без определения коэффициентов молярной экстинкции. Действительно, из закона Бэра и уравнения состояния идеального газа следует:

$$
p_{\text {InCl }}(T)=R T \frac{k(\lambda, T)}{\varepsilon(\lambda, T)} .
$$

Разумеется, отношение в правой части не зависит от длины волны. Если пренебречь температурной зависимостью экстинкции, то логарифм произведения $T k$ должен испытывать такую же температурную зависимость, как и $\ln p_{\text {InCl}}$, с точностью до зависящего от длины волны слагаемого:

$$
\ln T k(\lambda, T)=\ln p_{\mathrm{InCl}}(T)+\ln \frac{\varepsilon(\lambda)}{R} .
$$

Иначе говоря, в координатах $\ln T k-1 / T$ для различных полос поглощения насыщенного пара образуется набор прямых с одинаковым наклоном, отвечающим теплоте испарения расплава $\mathrm{InCl}^{*}$.

Закон Бера выполняется не только для коэффициента поглощения на данной (и любой) длине волны, но и для площади $S\left(\lambda_{1}, \lambda_{2}\right)$ под кривой $k(\lambda)$, то есть спектром поглощения, на интервале $\left(\lambda_{1}, \lambda_{2}\right)$. Действительно,

$$
S\left(\lambda_{1}, \lambda_{2}\right)=\int_{\lambda_{1}}^{\lambda_{2}} k(\lambda) d \lambda=E\left(\lambda_{1}, \lambda_{2}\right) C,
$$

где $E\left(\lambda_{1}, \lambda_{2}\right)=\int_{\lambda_{1}}^{\lambda_{2}} \varepsilon(\lambda) d \lambda$.

Практически интервал $\left(\lambda_{1}, \lambda_{2}\right)$ выбирается так, чтобы в него попала полоса поглощения или группа полос. Таким образом, вместо (3) имеем:

$$
p_{\mathrm{InCl}}(T)=R T \frac{S\left(\lambda_{1}, \lambda_{2} ; T\right)}{E\left(\lambda_{1}, \lambda_{2}\right)},
$$

и зависимости в координатах $\ln T S\left(\lambda_{1}, \lambda_{2}\right)-1 / T$ также должны спрямляться.

Как видно из рис. 4, экспериментальные данные по зависимостям для равновесия (1) хорошо аппроксимируются прямыми вида:

$$
\begin{aligned}
\ln T k(\lambda, T) & =-\frac{A}{T}+B(\lambda), \\
\ln T S\left(\lambda_{1}, \lambda_{2} ; T\right) & =-\frac{A}{T}+B^{\prime}\left(\lambda_{1}, \lambda_{2}\right),
\end{aligned}
$$

которые оказываются почти параллельными друг другу (линии 1-6 рис. 4). Параметры $A$ и $B$ сведены

* Это справедливо, конечно, если области гомогенности конденсированных фаз пренебрежимо малы или мало изменяются с температурой, а температурной зависимостью энтальпии парообразования $\mathrm{InCl}$ можно пренебречь. 
Табл. 1. Параметры зависимостей (5) и (6) для равновесия (1)

[Table 1. The parameters of dependencies (5) and (6) for equilibrium (1)]

\begin{tabular}{|c|c|c|c|c|c|}
\hline $\begin{array}{c}\text { № полосы } \\
\text { (рис. 4) } \\
\text { [Absorption } \\
\text { band number] }\end{array}$ & $\lambda, \mathrm{nm}$ & $N$ & $\begin{array}{c}\text { Вид } \\
\text { зависимости } \\
\text { [Type of } \\
\text { dependence] }\end{array}$ & $A, \mathrm{~K}$ & $\begin{array}{c}B \text { или } B^{\prime} \\
B \text { or } B^{\prime}\end{array}$ \\
\hline 1 & 266.2 & 24 & \multirow{4}{*}{ (5) } & $-10837 \pm 160$ & $25.58 \pm 0.29$ \\
\hline 2 & 267.0 & 17 & & $-9904 \pm 185$ & $24.78 \pm 0.35$ \\
\hline 3 & 270.4 & 24 & & $-9560 \pm 205$ & $22.73 \pm 0.37$ \\
\hline 4 & 272.6 & 27 & & $-11122 \pm 172$ & $24.07 \pm 0.29$ \\
\hline 5 & $262-280$ & 21 & \multirow{2}{*}{ (6) } & $-10609 \pm 99$ & $26.61 \pm 0.18$ \\
\hline 6 & $337-365$ & 14 & & $-10218 \pm 209$ & $22.42 \pm 0.29$ \\
\hline
\end{tabular}

$N$ - количество точек для обработки.

[N- number of processed points.]

Табл. 2. Параметры температурной зависимости давления насыщенного пара монохлорида индия в равновесии (1)

[Table 2. The parameters of the temperature dependence of saturated vapor pressure of indium monochloride in equilibrium (1)]

\begin{tabular}{|c|c|c|c|}
\hline \multirow{2}{*}{$\begin{array}{c}\text { Ссылка } \\
\text { [Reference] }\end{array}$} & \multicolumn{2}{|c|}{$\begin{array}{c}\text { Параметры уравнения (7) } \\
\text { [Parameters of equation (7)] }\end{array}$} & $\begin{array}{c}\text { Интервал } \\
\text { температуры, K } \\
\text { [Temperature range / K] }\end{array}$ \\
\cline { 2 - 3 } & $A, \mathrm{~K}$ & $b$ & $643-927$ \\
\hline$[15]$ & -10546 & 11.32 & $573-863$ \\
\hline$[16]$ & -10668 & 11.85 & $583-713$ \\
\hline$[17]$ & -10659 & 11.50 & $729-924$ \\
\hline$[18]$ & -10182 & 10.99 & $980-1090$ \\
\hline$[19]$ & -9793 & 10.53 & $828-1012$ \\
\hline $\begin{array}{c}\text { наши данные } \\
\text { [this work] }\end{array}$ & $-10255 \pm 69$ & $10.95 \pm 0.08$ & \\
\hline
\end{tabular}

Давление в уравнении (7) дано относительно стандартного $p_{0}=1$ атм.

[The pressure in equation (7) is given relative to the standard $p_{0}=1 \mathrm{~atm}$.]

в табл. 1, а в табл. 2 представлены литературные данные [15-19] по температурной зависимости давления пара в равновесии (1). Угловые коэффициенты, полученные для разных длин волн, мало отличаются друг от друга и близки угловым коэффициентам табл. 2. Таким образом, показана взаимная корреляция спектрофотометрических и литературных тензиметрических данных по рассматриваемому равновесию.

Вместе с тем, различие угловых коэффициентов, найденных для различных полос поглощения (табл. 1), не укладывается в доверительные интервалы, получаемые при обработке данных по методу наименьших квадратов. На наш взгляд, такое расхождение происходит вследствие заметного и притом различного влияния температуры на экстинкцию разных полос поглощения пара InCl. По этой причине влияние температуры на светопоглощение учитывалось нами далее в ходе определения коэффициентов молярной экстинкции.

\section{РЕЗУЛЬТАТЫ НУЛЬ-МАНОМЕТРИЧЕСКОГО ЭКСПЕРИМЕНТА}

Для решения главной поставленной в работе задачи - нахождения коэффициентов молярной экстинкции $\mathrm{InCl}$ для различных полос поглощения, - необходимы данные прямого определения давления в равновесии (1). Для этого использовали кварцевый мембранный нуль-манометр, а измерение давления компенсирующего газа - аргона, проводили при помощи пьезорезистивных датчиков давления MPX-5050 и MPX-4115 (Motorola), позволяющих измерять давления от $10^{2}$ и $10^{3} \mathrm{~Pa}$ до $5.0 \cdot 10^{4}$ и $2.3 \cdot 10^{5} \mathrm{~Pa}$ соответственно. Погрешность измерения для этих датчиков соответствовала нижней границе диапазона давления. Показания датчиков проверялись по ртутному манометру, конструкция которого описана в [20, с. 130-167]. Если расхождения между показаниями датчиков и манометра не превышали 400 Ра, то предпочтение отда- 
валось показаниям датчиков; в противном случае измерение проводилось повторно после вакуумирования датчиков. Методики подготовки и проведения манометрического эксперимента подробно описаны в [21].

Измерения давления производились в диапазоне температур от 220 до $739{ }^{\circ} \mathrm{C}$. Полученные данные по температурной зависимости давления пара представлены на рис. 6. Данные для температур 555-739 ${ }^{\circ} \mathrm{C}$ (соответствующие давлениям $2.49 \cdot 10^{4}-2.23 \cdot 10^{5} \mathrm{~Pa}$, всего 41 точка) описывались уравнением:

$$
\ln p_{\mathrm{InCl}}=-\frac{A}{T}+b
$$

Здесь используется давление в относительных единицах, то есть отношение абсолютного измеренного давления к стандартному (реперному), в качестве которого было взято давление $p_{0}=1 \mathrm{~atm}$. Таким образом, величина, стоящая слева под логарифмом, безразмерна*. Полученные коэффициенты $A$ и $b$ даны в табл. 2 в сравнении с литературными данными.

Заметим, что в работах [15-19] сообщалось об исследовании равновесия $L_{\mathrm{InCl}}-V$, причем в качестве исходного вещества был взят кристал-

* То же относится ко всем логарифмическим уравнениям, в частности, (5) и (6).

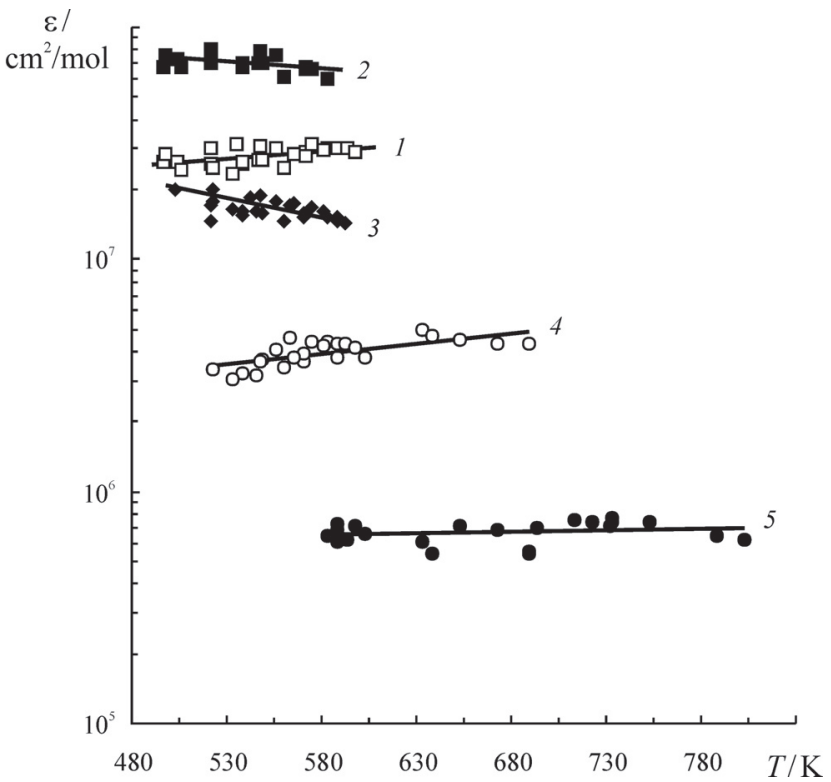

Рис. 5. Температурные зависимости коэффициента молярной экстинкции пара $\mathrm{InCl}$ на длинах волн: 1 266.2, 2 - 267.0, 3 - 270.4, 4-272.6, 5-342.4 nm

[Fig. 5. The temperature dependences of the molar extinction coefficient of indium chloride vapor for the bands: 1 - 266.2, 2-267.0, 3-270.4, 4 - 272.6, 5 $342.4 \mathrm{~nm}]$ лический $\mathrm{InCl}$, который по данным [6] разлагается при $\sim 220^{\circ} \mathrm{C}$ по синтектической реакции $S_{\mathrm{InCl}} \rightarrow L_{\mathrm{In}}+L^{\prime}$, где $L^{\prime}-$ жидкость, обогащенная хлором. Характер фазовой диаграммы системы In-Cl однако таков, что, с одной стороны, растворимость хлора в жидком индии чрезвычайно мала, и, с другой, состав жидкости $L^{\prime}$ практически совпадает со стехиометрическим составом $\mathrm{InCl}$ (символически: $\left.L^{\prime}=L_{\mathrm{InCl}}\right)$ - кривая купола расслоения вертикальна на значительном участке температур. С учетом этого мы полагали, что в цитируемых работах в действительности исследовалось равновесие (1).

Манометрические данные использовались далее для нахождения коэффициентов молярной экстинкции $\mathrm{InCl}$ для максимумов полос поглощения при различных температурах.

I способ. Принимая, что для рассматриваемого равновесия общее давление $p$ ввиду существования в паре единственной формы InCl совпадает с парциальным давлением $p_{\text {InCl}}$, экстинкцию можно вычислить непосредственно из уравнения (3). Если вместо коэффициента поглощения использовать площадь под полосой (или группой полос) $S\left(\lambda_{1}, \lambda_{2}\right)$, то полученное значение соответствует интегральному коэффициенту $E\left(\lambda_{1}, \lambda_{2}\right) * *$. Для расчета давления насыщенного пара $p_{\mathrm{InCl}}(T)$ использовали данные последней строки табл. 2, поскольку они гарантировано отвечают равновесию (1) (см. примечание 2 к табл. 2).

На рис. 5 представлены некоторые рассчитанные для разных температур значения $\varepsilon(\lambda)$ и $E\left(\lambda_{1}, \lambda_{2}\right)$. Как и ожидалось, исходя из предыдущих результатов, эти величины не сильно, но заметно изменяются с температурой, причем наблюдаемое изменение линейно:

$$
\varepsilon(\lambda, T)=\alpha(\lambda)+\beta(\lambda) T, \quad E(\lambda, T)=\alpha^{\prime}(\lambda)+\beta^{\prime}(\lambda) T .
$$

Параметры таких зависимостей приведены в табл. 3, в которой в качестве примера даны также величины коэффициентов молярной экстинкции, рассчитанные для температуры $327^{\circ} \mathrm{C}$.

II способ. Для некоторых полос поглощения удалось провести независимую проверку полученных величин экстинкции при использовании результатов эксперимента со сменой характера равновесия (1)-(2). Для этого участки температурной зависимости $k(\lambda, T)$, относящиеся к равновесию (1) (кривая $a b$ рис. 2) аппроксимировали уравнением

** Заметим, что площадь $S$ безразмерна, ибо размерность коэффициента поглощения $[k]=L^{-1}$. Следовательно, коэффициент $E$ имеет размерность $[E]=L^{3} / N$, обратную размерности молярной объемной концентрации. В настоящей работе эту величину выражали в $\mathrm{cm}^{3} / \mathrm{mol}$. 
Табл. 3. Параметры температурной зависимости и значения при $323{ }^{\circ} \mathrm{C}$ коэффициента молярной экстинкции пара $\mathrm{InCl}$

[Table 3. The parameters of the temperature dependence and values at $323{ }^{\circ} \mathrm{C}$ of the molar extinction coefficient of vapor $\mathrm{InCl}]$

\begin{tabular}{|c|c|c|c|c|}
\hline \multirow[b]{2}{*}{$\lambda, \mathrm{nm}$} & \multirow[b]{2}{*}{$\begin{array}{c}\alpha, \\
\mathrm{cm}^{2} / \mathrm{mol}\end{array}$} & \multirow[b]{2}{*}{$\begin{array}{c}\beta, \\
\mathrm{cm}^{2} /(\mathrm{mol} \cdot \mathrm{K})\end{array}$} & \multicolumn{2}{|c|}{$\varepsilon(\lambda), \mathrm{cm}^{2} / \mathrm{mol}$} \\
\hline & & & $\begin{array}{c}\text { I способ } \\
\text { [I method] }\end{array}$ & $\begin{array}{c}\text { II способ } \\
\text { [II method] }\end{array}$ \\
\hline 266.2 & $4.299 \cdot 10^{6}$ & $4.321 \cdot 10^{4}$ & $3.02 \cdot 10^{7}$ & - \\
\hline 267.0 & $1.167 \cdot 10^{8}$ & $-8.651 \cdot 10^{4}$ & $6.48 \cdot 10^{7}$ & - \\
\hline 270.4 & $3.822 \cdot 10^{7}$ & $-3.918 \cdot 10^{4}$ & $1.47 \cdot 10^{7}$ & $1.50 \cdot 10^{7}$ \\
\hline 272.6 & $-1.284 \cdot 10^{6}$ & $9.114 \cdot 10^{3}$ & $4.18 \cdot 10^{6}$ & $4.28 \cdot 10^{6}$ \\
\hline 342.4 & $5.240 \cdot 10^{5}$ & $2.148 \cdot 10^{2}$ & $6.53 \cdot 10^{5}$ & - \\
\hline $\begin{array}{c}\text { Интервал } \\
\text { [Range] } \\
\lambda_{1}-\lambda_{2}(\mathrm{~nm})\end{array}$ & $\begin{array}{c}\alpha^{\prime} \\
\mathrm{cm}^{3} / \mathrm{mol}\end{array}$ & $\begin{array}{c}\beta^{\prime}, \\
\mathrm{cm}^{3} /(\mathrm{mol} \cdot \mathrm{K})\end{array}$ & \multicolumn{2}{|c|}{$E\left(\lambda_{1}, \lambda_{2}\right), \mathrm{cm}^{3} / \mathrm{mol}$} \\
\hline $262-280$ & $5.070 \cdot 10^{7}$ & $1.245 \cdot 10^{5}$ & \multicolumn{2}{|c|}{$1.25 \cdot 10^{8}$} \\
\hline $337-365$ & $2.283 \cdot 10^{6}$ & $1.348 \cdot 10^{3}$ & \multicolumn{2}{|c|}{$3.09 \cdot 10^{6}$} \\
\hline
\end{tabular}

(5), линейным в координатах $\ln T k(\lambda)-1 / T$, а участок, соответствующий равновесию (2) (кривая ef рис. 2), - также линейной зависимостью от температуры, но в координатах $k(\lambda)-T$. Последнюю зависимость экстраполировали в область низких температур и искали точку пересечения линии $a b$ и ef (точка $d$ на рис. 2), в которой смена вариантности равновесия происходила бы в условиях пренебрежимо малой растворимости хлора в расплаве металлического индия. Координаты этой точки получали при совместном (численном) решении аппроксимирующих уравнений для линий $a b$ и $e f$. Для описываемого эксперимента такое решение дало температуру $t_{d}=327^{\circ} \mathrm{C}$.

Поскольку точка $d$ принадлежит равновесию (1), уравнение (3) позволяет найти величину коэффициента молярной экстинкции $\varepsilon(\lambda)$ по известным величинам $k_{d}(\lambda)$ и $T_{d}$ при учете температурной зависимости давления пара в этом равновесии. В свою очередь, в равновесии (2) в расчет принимались данные, полученные при значительно более высоких температурах, чем при обработке результатов по первому способу. Тогда, если бы величина $\varepsilon(\lambda)$ или ее температурная зависимость находились с большой ошибкой, то наблюдалось бы сильное рассогласование результатов, рассчитываемых по первому и второму способам.

Заметим, что в настоящей работе, к сожалению, проводился только один эксперимент со сменой характера равновесия, и сравнить величины $\varepsilon$, получаемые по первому и второму способам, оказалось возможным только для единственной температуры $T_{d}$. Однако и такое сравнение оказалось информативным. Как видно из табл. 3 (две последние колон- ки), полученные по первому и второму способам величины $\varepsilon$ почти одинаковы. Это указывает на то, что температурная зависимость коэффициента молярной экстинкции в исследованных пределах корректно описывается как линейная. Проверку удалось провести только для экстинкции при длинах волн 270.4 и $272.6 \mathrm{~nm}$. Для полос с максимумами 266.2 и $267.0 \mathrm{~nm}$ поглощение оказалось слишком сильным, а для полосы 342.4 nm, напротив, слабым для определения величин $k$ и $\varepsilon$.

Еще одна проверка, позволяющая видеть согласование данных, была проведена при обратном вычислении давления насыщенного пара InCl c помощью уравнений (3) и (4), в котором использованы экспериментальные значения $k(\lambda)$ и найденные по температурным зависимостям значения $\varepsilon(\lambda)$ для разных длин волн (табл. 3$)$. Полученные результаты, представленные на рис. 6, попадают практически на одну прямую линию в координатах $\ln p_{\mathrm{InCl}}-1 / T$. Действительно, если значения $k$ или температурные зависимости $\varepsilon$ были получены с большой ошибкой, то не удалось бы получить наблюдаемую на рис. 6 хорошую корреляцию результатов, которые относятся к разным полосам поглощения и к площадям под этими полосами.

\section{ЗАКЛЮЧЕНИЕ}

1. Получены электронные абсорбционные спектры пара монохлорида индия в широком диапазоне температур. Подтверждено мнение авторов [68] о том, что в равновесии пара хлоридов индия с расплавами металлического индия в паре присутствует практически только монохлорид. 


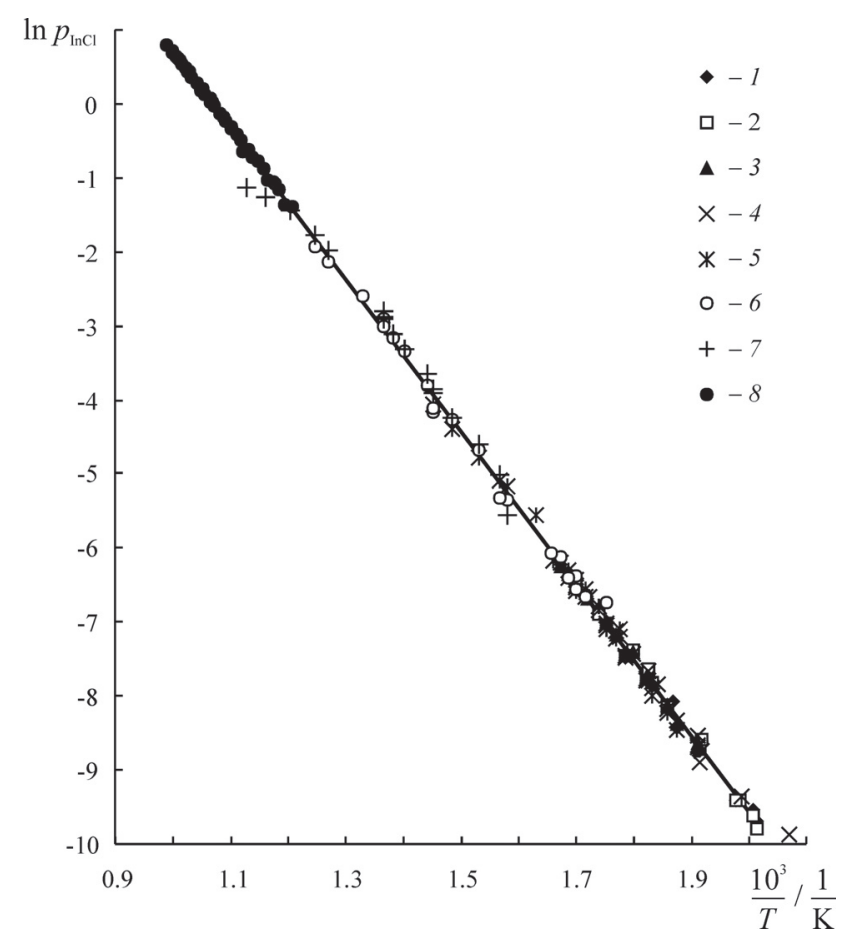

Рис. 6. Температурная зависимость давления насыщенного пара $\mathrm{InCl}$ в равновесии (1), вычисленного из спектрофотометрических данных при длинах волн: 1 - 266.2; 2 - $267.0 \mathrm{~nm} ; 3$ - интервал 262 - $280 \mathrm{~nm}$ (по площади); 4 - 270.4; 5 - 272.6; 6 - 342.4 nm; 7 интервал 337 - 365 nm (по площади); 8 - манометрические данные настоящей работы

[Fig. 6. The temperature dependence of the saturated vapor pressure of $\mathrm{InCl}$ in equilibrium (1), calculated from spectrophotometric data for wavelengths: 1 - 266.2; 2 $267.0 \mathrm{~nm} ; 3$ - range 262 - $280 \mathrm{~nm}$ (by area); $4-270.4$; 5 - 272.6; 6 - $342.4 \mathrm{~nm} ; 7$ - range $337-365 \mathrm{~nm}$ (by area); 8 - manometric data of this work]

2. В ходе тензиметрического эксперимента с использованием кварцевого мембранного нуль-манометра определена температурная зависимость давления пара в трехфазном равновесии между расплавом металлического индия, расплавом хлорида индия и паром. Показано, что эта зависимость хорошо коррелирует с литературными данными.

3. С использованием результатов спектрофотометрических и тензиметрических экспериментов определены величины коэффициентов молярной экстинкции для наиболее сильных полос поглощения пара монохлорида индия. Полученные значения (табл. 3) показывают, что светопоглощение пара InCl для группы полос в диапазоне длин волн 262 - $280 \mathrm{~nm}$ является одним из самых мощных среди газов в области «воздушного» УФ и видимого излучения. Наибольшее значение коэффициента молярной экстинкции найдено для полосы с максимумом поглощения $267.0 \mathrm{~nm}$ и составляет $1.17 \cdot 10^{8} \mathrm{~cm}^{2} / \mathrm{mol}$ при $327{ }^{\circ} \mathrm{C}$ (что, например, в 150 раз больше молярной экстинкции $\mathrm{I}_{2}$ в паре на длине волны $520 \mathrm{~nm})$. Такое интенсивное светопоглощение позволяет фиксировать присутствие $\mathrm{InCl}$ в паре при парциальных давлениях от десятых долей Па, что соответствует концентрациям $\sim 1 \cdot 10^{-10} \mathrm{~mol} / \mathrm{cm}^{3}$. Таким образом, спектрофотометрический метод оказывается очень чувствительным для количественного определения данной молекулярной формы.

С другой стороны, высокая абсорбция монохлорида индия может мешать исследованиям, когда ничтожная примесь этого хлорида ошибочно принимается за другие молекулярные формы летучих соединений индия. Так, в [10] сообщалось об ошибочном определении формы In 2 Se в паре над селенидами индия в работе [22] этих же авторов, а также отмечалась чрезвычайная сложность глубокой очистки индия и селенидов индия от примеси InCl при проведении спектрофотометрических исследований систем, в которых хлор является посторонним компонентом.

4. Высокие величины коэффициентов молярной экстинкции паров монохлорида индия для его самых мощных полос поглощения ограничивают верхний предел спектрофотометрического измерения давления пара этого вещества. Так, при давлениях уже чуть выше $60 \mathrm{~Pa}$, коэффициент поглощения при $267.0 \mathrm{~nm}$ превышает $5 \mathrm{~cm}^{-1}$ и, следовательно, измеряется с высокой погрешностью. Однако использование данных для менее мощных полос поглощения (с длинами волн 270.6, 272.4, 342.4 nm и др.) позволяет значительно расширить верхние пределы измерений и определять давление паров $\mathrm{InCl}$ вплоть до атмосферного.

5. Показано, что при проведении спектрофотометрических исследованиях пара в изотермическом эксперименте целесообразно учитывать температурную зависимость коэффициентов молярной экстинкции. (На необходимость учета такой зависимости при проведении оптико-тензиметрических экспериментов указано, например, в известной монографии [23]). Обнаружено, что даже для близкорасположенных полос поглощения может наблюдаться различный ход температурных зависимостей $\varepsilon(\lambda, T)$, когда для одних полос эти значения увеличиваются с температурой, а для других уменьшаются. Анализ этого явления выходит, однако, за рамки данной работы.

6. Хорошее согласование значений углового коэффициента $A$, полученных с помощью нуль- 
манометрического (уравнение (7)) и спектрофотометрического (уравнения $(5,6))$ методов, позволяет рассматривать высокотемпературную спектрофотомерию пара как альтернативу прямому манометрическому эксперименту. При относительно более простой постановке опыта фотометрия пара дает возможность не только исследовать качественный и количественный состав, что является обычной химико-аналитической задачей, но и получать количественные данные о фазовых равновесиях с участием пара, в частности, теплоты испарения конгруэнтно испаряющихся конденсированных фаз.

\section{ИСТОЧНИК ФИНАНСИРОВАНИЯ}

Работа выполнена при финансовой поддержке РФФИ, проект 18-33-00900-мол-а.

\section{КОНФЛИКТ ИНТЕРЕСОВ}

Авторы декларируют отсутствие явных и потенциальных конфликтов интересов, связанных с публикацией настоящей статьи.

\section{СПИСОК ЛИТЕРАТУРЫ}

1. Sen D., Heo N., Sef K. // J. Phys. Chem. C, 2012, vol. 116 , no. 27 , pp. $14445-14453$. DOI: $10.1021 /$ jp303699u

2. Kitsinelis S., Zissis G., Fokitis E. // J. Physics D: Appl. Phys. 2009, vol. 42, p. 045209 (8pp). DOI: 10.1088/0022-3727/42/4/045209

3. Hayashi D., Hilbig R., Kurber A., et. al. // Appl. Phys. Letters, 2010, vol. 96, p. 061503. DOI: 10.1063/ 1.3318252

4. Binnewies M., Schmidt M., Schmidt P. // Z. Anorg. Allg. Chem. 2017, vol. 643, pp. 1295-1311. DOI: 10.1002/ zaac. 201700055

5. Zavrazhnov A. Y., Turchen D. N., Naumov A. V., Zlomanov V. P. // J. Phase Equilibria. 2003, vol. 24, no. 4, pp. 330-339. DOI: $10.1361 / 105497103770330316$

6. Федоров П. И., Акчурин Р. Х. Индий. М.: Наука, $2000,276 \mathrm{c}$.

7. Zavrazhnov A. Yu., Naumov A. V., Pervov V. S., Riazhskikh M. V. // Thermochimica Acta, 2012, vol. 532, pp. 96-102. DOI:10.1016/j.tca.2010.10.004
8. Завражнов А. Ю., Наумов А. В., Сергеева А. В., Сидей В. И. // Неорганические материальь, 2007, vol. 43, no. 11, pp. 1303-1315. DOI: 10.1134/ S0020168507110039

9. Завражнов А. Ю., Косяков А. В., Сергеева А. В., Березин С. С., Черненко К. К. // Конденсированные среды и межфазные гранищы, 2017, т. 17, № 4, с. 417436. URL: https://journals.vsu.ru/kcmf/article/ view/87/190

10. Brebrick R. F. // J. Phase Equilibria and Diffusion, 2005 , vol. 26 no. 1 , pp. $20-21$. DOI: $10.1007 /$ s11669-0050054-z

11. Kuniga Y., Hosaka M. // J. Cryst. Growth, 1975, vol. 28, pp. 385-391. DOI: 10.1016/0022-0248(75)90077-9

12. Froslie H. M., Winans J. G. // Phys. Rev., 1947, vol. 72 , iss. 6 , pp. 481-491. DOI: /doi.org/10.1103/physrev. 72.481

13. Jones W. E., McLean T. D. // J. Molecular Spectroscopy, 1991, vol. 150, iss. 1, pp. 195-200. DOI: 10.1016/00222852(91)90202-L

14. Vempati S. N., Jones W. E. // J. Molecular Spectroscopy, v. 132, iss. 2, pp. 458-466. DOI: 10.1016/00222852(88)90339-6

15. Kunia Y., Hosada S., Hosuka M. // Denki Kagaku Technical Paper, 1974, vol. 42, pp. 20-25.

16. Robert C. // Helv. Phys. Acta, 1936, vol. 9, pp. 405436.

17. Федоров П. И., Мохосоев М. В. Химия галлия, индия и таллия. Новосибирск, Наука, 1977, 224 с.

18. Дриц М. Е., Будберг П. Б., Бурханов Г. С. и др. Свойства элементов. Справочник под ред. М. Е. Дрица. М.: Металлургия, 1985, 672 с.

19. Бронников А. Д., Василевская И. И., Нисельсон Л. А. // Изв. АН СССР, Металлы, 1974, № 4, c. $54-57$.

20. Завражнов А. Ю. Дисс. ... докт. хим. наук. Воронеж, 2004, 340 c.

21. Завражнов А. Ю. // Журн. Неорган. Химии, 2003, т. 48 , №. 10 , c. $1722-1736$.

22. Brebrick R. F., Su C.-H. // J. Phase Equilibria, 2002, vol. 23, 2002, pp. 397-408. DOI: 10.1361/1054971027703 31343

23. Суворов А. В. Термодинамическая химия парообразного состояния. Л.: Химия, 1970, 208 с. 


\title{
THE PRESSURE OF INDIUM MONOCHLORIDE VAPOUR: THE VAPOUR-GAUGE AND SPECTROPHOTOMETRIC EXPERIMENTAL DATA
}

\author{
(C) 2019 A. Yu. Zavrazhnov*, A. V. Naumov, E. N. Malygina, A. V. Kosyakov \\ Voronezh State University \\ 1, Universitetskaya pl., 394018 Voronezh, Russian Federation
}

\begin{abstract}
Equilibria involving indium and gallium halides are important, in particular, for the deep purification of the metallic indium and gallium. At the same time, while gallium can be easily transported during the halide CVT, a similar indium transfer is practically impossible. The following study was carried out to as an endeavour to identify the causes of this difficulty.

Objective. The goal of the research was to conduct the spectrophotometric and vapour-pressure investigation of the two-phase $L_{\text {In }}-V$ and the three-phase $L_{\text {In }}-L_{\text {InCl }}-V$ equilibria and to find the molar extinction coefficients of gaseous indium monochloride. ( $L_{\mathrm{In}}$ and $L_{\mathrm{InCl}}$ are liquids based on metallic In and $\mathrm{InCl}$ respectively).

Methods and methodology. To investigate the above-mentioned equilibria, the following optical vapour pressure methods were used: pressure gauge technique and high-temperature spectrophotometry (MDR-41 monochromator, combined with the cylindrical furnace). Electronic absorption spectra of indium monochloride vapour were obtained in the wavelength range of $200-400 \mathrm{~nm}$ and a temperature range of $225-850^{\circ} \mathrm{C}$.

Results. The spectrophotometric studies of the $L_{\text {In }}-V$ equilibrium allowed us to show the temperature dependence of the absorption coefficients at wavelengths corresponding to the absorption bands maxima under conditions when the indium monochloride concentration in a vapour remained constant. It was shown that in the $L_{\mathrm{In}}-V$ equilibrium the concentration $C_{\mathrm{InCl}}=$ const and the change in the absorption coefficients could only be associated with the temperature dependence of the InCl extinction. The fact is that the characteristic absorption bands of other possible species, $\mathrm{In}_{2} \mathrm{Cl}_{4}$ and $\mathrm{InCl}_{3}$, were not observed. Therefore, we can assume that only $\mathrm{InCl}$ molecules are present in the vapour in the $L_{\text {In }}-V$ equilibrium.

The experimental dependences of the absorption coefficient $k(\lambda, T)$ of the $\mathrm{InCl}$ saturated vapour (in the three-phase equilibrium $L_{\mathrm{In}}-L_{\mathrm{InCl}}-V$ ) on the temperature can be described by the following function:

$$
\ln T k(\lambda, T)=-\frac{A}{T}+B(\lambda) .
$$

It should be noted that the angular coefficient $A$ is essentially independent of the wavelength and its value is almost identical to the angular coefficient for the temperature dependence of the pressure of saturated vapour:

$$
\ln p=-\frac{A}{T}+b .
$$

The latter dependency was found in the course of the vapour pressure experiments for the three-phase equilibrium with the use of a quartz membrane null-manometer.

Conclusion. For the latter equation the following parameters were obtained: $A=-10255 \pm 69 \mathrm{~K}$, $b=1095 \pm 0.08$ (for the atmospheric pressure as a standard for $p$ ). These values correlate well with the reported data. These results were used to calculate the molar extinction coefficients for the strongest absorption bands of gaseous indium monochloride. The highest value of the molar extinction coefficient $\varepsilon(\lambda)$ was found for the band at $267.0 \mathrm{~nm}$ which is $1.17 \cdot 10^{8} \mathrm{~cm}^{2} / \mathrm{mol}$ at the temperature of $327^{\circ} \mathrm{C}$. For other bands in the range of $262-280 \mathrm{~nm}$ the $\varepsilon(\lambda)$-values are also very high. Thus, the spectrophotometric method is very sensitive for both qualitative and quantitative determination of gaseous indium monochloride.
\end{abstract}

Keywords: indium monochloride, saturated vapour pressure, heterogeneous equilibrium, high temperature spectrophotometry, tensimetry, manometry.

$\bar{\square}$ Zavrazhnov Alexander Yu., e-mail: alzavr08@rambler.ru 


\section{SOURCE OF FINANCING}

The reported study was supported by the Russian Foundation for Basic Research, (project No.18-3300900- mol-a)..

\section{CONFLICT OF INTEREST}

The authors declare the absence of obvious and potential conflicts of interest related to the publication of this article.

\section{REFERENCES}

1. Sen D., Heo N., Sef K. J. Phys. Chem. C, 2012, vol. 116, no. 27, pp. 14445-14453. DOI: 10.1021/jp303699u

2. Kitsinelis S., Zissis G., Fokitis E. J. Physics D: Appl. Phys., 2009, vol. 42, p. 045209 (8pp). DOI: 10.1088/0022$3727 / 42 / 4 / 045209$

3. Hayashi D., Hilbig R., Kurber A., et al. Appl. Phys. Letters, 2010, vol. 96, p. 061503. DOI: 10.1063/1.3318252

4. Binnewies M., Schmidt M., Schmidt P. Z. Anorg. Allg. Chem., 2017, vol. 643, pp. 1295-1311. DOI: $10.1002 /$ zaac. 201700055

5. Zavrazhnov A. Y., Turchen D. N., Naumov A. V., Zlomanov V. P. J. Phase Equilibria., 2003, vol. 24, no. 4, pp. 330-339. DOI: $10.1361 / 105497103770330316$

6. Fedorov P. I., Akchurin R. Kh. Indium. Moscow, Nauka Publ., 2000, 276 p. (in Russ.)

7. Zavrazhnov A. Yu., Naumov A. V., Pervov V. S., Riazhskikh M. V. Thermochimica Acta, 2012, vol. 532, pp. 96-102. DOI:10.1016/j.tca.2010.10.004

8. Zavrazhnov A. Yu., Naumov A. V., Sergeeva A. V., Sidei V. I. Inorganic Materials (Russia), 2007, vol. 43, no. 11, pp. 1167-1178. DOI: 10.1134/S0020168507110039

9. Zavrazhnov A. Yu, Kosyakov A. V, Sergeeva A. V., Berezin S. S. Condensed Matter and Interphases, vol. 17, no. 4 , pp. $417-436$. URL: https://journals.vsu.ru/kcmf/ article/view/87/190 (in Russ.)
10. Brebrick R. F. J. Phase Equilibria and Diffusion, 2005, vol. 26 no. 1 , pp. 20 - 21. DOI: 10.1007/s11669-005-0054-z

11. Kuniga Y., Hosaka M. J. Cryst. Growth, 1975, vol. 28, pp. 385-391. DOI: 10.1016/0022-0248(75)90077-9

12. Froslie H. M., Winans J. G. Phys. Rev., 1947, vol. 72 , iss. 6, pp. 481-491. DOI: /doi.org/10.1103/physrev.72.481

13. Jones W. E., McLean T. D. J. Molecular Spectroscopy, 1991, vol. 150, iss. 1, pp. 195-200. DOI: 10.1016/00222852(91)90202-L

14. Vempati S. N., Jones W. E. J. Molecular Spectroscopy, vol. 132 , iss. 2 , pp. $458-466$. DOI: $10.1016 / 0022-$ 2852(88)90339-6

15. Kunia Y., Hosada S., Hosuka M. Denki KagakuTechnical Paper, 1974, vol. 42, pp. 20-25.

16. Robert C. Helv. Phys. Acta, 1936, vol. 9, pp. 405436.

17. Fedorov P. I., Mokhosoyev M. V. Gallium, Indium and Thallium Chemistry. Novosibirsk, Nauka Publ., 1977, 224 p. (in Russ.)

18. Dritz M. E., Budberg P. ., Burkhanov G. S., et al. Properties of the Elements. Handbook, ed. by Dritz M. E. Moscow, Metallurgia Publ., 1985, 672 p. (in Russ.)

19. Bronnikov A. D., Valilevskaya I. I., Niselson L. A. Izv. AN. SSSR. Metally, 1974, no. 4, pp. 54-57. (in Russ.)

20. Zavrazhnov A. Yu. Diss. Doct. chem. sci. Voronezh, 2004,340 p.

21. Zavrazhnov A. Yu. Russian Journal of Inorganic Chemistry, 2003, vol. 48, no. 10, pp. 1577-1590. (in Russ.)

22. Brebrick R. F., Su C.-H. J. Phase Equilibria, 2002, vol. 23, 2002, pp. 397-408. DOI: 10.1361/1054971027703 31343

23. Suvorov A. V. Thermodynamicheskaya chimia paroobraznogo sostoyania [Thermodynamic Chemistry Vapor State]. Leningrad, Chimia Publ., 1970, 208 p. (in Russ.)

Zavrazhnov Alexander Yu. - Dr. Sci. (Chem.), Professor, Department of General and Inorganic Chemistry, Voronezh State University, Voronezh, Russian Federation; e-mail: alzavr08@rambler.ru. ORCID: 00000003-0241-834X.

Naumov Alexander V. - Cand. Sci. (Chem.), Assistant Professor, Department of General and Inorganic Chemistry, Voronezh State University, Voronezh, Russian Federation; e-mail: aither@bk.ru. ORCID: 00000002-1313-8573.

Malygina Ekaterina $N .-5^{\text {rd }}$ year Student at Faculty of Chemistry, Voronezh State University, Voronezh, Russian Federation; e-mail: ekaterina.malygina2013@ yandex.ru. ORCID: 0000-0001-7179-335X.

Kosyakov Andrew V. - Cand. Sci. (Chem.), Assistant Professor, Department of General and Inorganic Chemistry, Voronezh State University, Voronezh, Russian Federation; e-mail: lavchukb@mail.ru. ORCID: 0000-0001-9662-7091. 